\title{
Glycolysis Induction
}

National Cancer Institute

\section{Source}

National Cancer Institute. Glycolysis Induction. NCI Thesaurus. Code C40634.

Glycolysis Induction involves initiation of activities of the pathway by which glucose is catabolized into two molecules of pyruvic acid with the generation of ATP. 